\title{
An AutoPilot platform for high-resolution light-sheet microscopy
}

\begin{abstract}
A framework for adaptive light-sheet microscopy allows experts and non-experts alike to sit back, relax, and capture the best images their systems can provide.

So many things in our lives are becoming autonomous and 'smart' - smart cars, smart phones, smart watches. Loïc Royer and Philipp Keller want to make microscopes smart. With recent developments in imaging technologies, like light-sheet microscopy (LSM), their timing couldn't be better.

"For live imaging experiments, lightsheet microscopy allows you to see the entire system; be it an entire embryo, or tens of thousands of neurons as they contribute to computations instructive to animal behavior", says Keller, a Group Leader at the Janelia Research Campus, Howard Hughes Medical Institute. Additionally, LSM is significantly gentler on living tissue than conventional fluorescence microscopy, enabling long-term in vivo imaging of creatures as they develop and grow.
\end{abstract}

But as these complex samples change over time, so too do their optical properties, which can decrease LSM image quality. As Keller explains, "You may start with something that looks good, but over time it gets worse and worse, and you realize you are losing control. The assumptions at the beginning of the experiment don't hold anymore."

The complexities of LSM, which uses a dedicated illumination system and a separate detection system, make it unfeasible to continuously optimize image quality by hand; the manual twisting of knobs most microscope users are accustomed to just won't cut it. So Keller teamed up with Royer, a postdoc at the Max Planck Institute of Molecular Cell Biology and Genetics, and other team members to develop an automated solution. Royer, with a background in physics and engineering and some previous experience automating simpler microscope systems, jumped right in. "The first thing I noticed with light-sheet microscopes is that there are lots of degrees of freedom. So we developed a framework
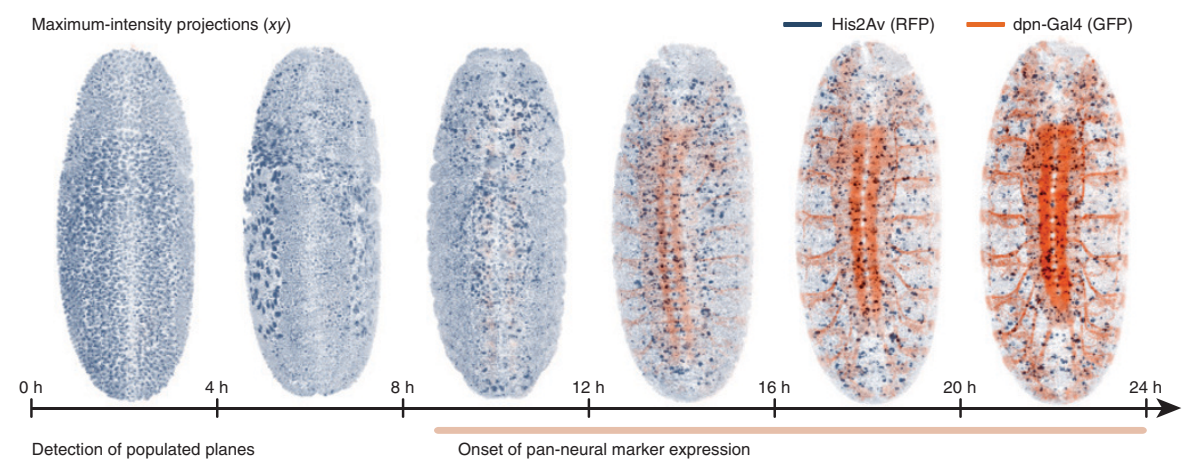

Max-intensity projections of labeled cells in a $D$. melanogaster developing embryo using light-sheet microscopy and the AutoPilot platform. Image adapted from Nat. Biotech. 34, 1267-1278; 2016.

that incorporates algorithms and also a mathematical model to describe the microscope and the relationships between all of its parts and how they affect image quality."

The resulting automation method, called 'AutoPilot', makes use of the down-time between collecting image stacks to gather information about the sample and make any adjustments to several degrees of freedom for controlling illumination and detection planes of the light-sheet microscope (Nat. Biotech. 34, 1267-1278; 2016). Importantly, the AutoPilot feedback to the microscope is rapid and requires only an additional $5 \%$ of light exposure to the sample, allowing optimization without hindering LSM's core benefits of fast and gentle whole-system imaging. Using developing zebrafish and drosophila embryos, as well as whole-brain functional imaging in zebrafish larvae, Royer et al. demonstrate AutoPilot's ability to automate image optimization and significantly improve resolution and signal strength.

The team hopes the AutoPilot platform will serve as a flexible foundation for people to build on as imaging technologies continue to expand. Likewise, they anticipate it will help level the playing field between expert and non-expert microscope users, allowing non-experts to reap the benefits of increasingly complex microscope systems. "Making microscopes more powerful means, to some extent, making them more complex. AutoPilot can help take away some of the complexities for the user... ensuring you have optimal image quality" says Keller. His group has already incorporated the AutoPilot platform into all of the lab's microscopes. “There's simply no good reason for us not to use it in every experiment we are doing."

Royer also sees the potential for the AutoPilot platform to scale-up image-based phenotyping capabilities and drug screening for pharmaceutical companies. "For example, if you're a drug company that wants to test compounds on tissue regeneration in zebrafish, you would need to be able to test these compounds on hundreds of samples at the same time." By providing better images and higher-throughput, AutoPilot could be an important automation tool for industry to add to existing protocols. But, regardless of any specific uptake of the AutoPilot framework, Royer and Keller hope anyone in the scientific community can use and improve on the platform, and have made the code for the framework freely available. As Royer comments, "We did our best to put the ideas out there so that companies and researchers can add this platform to their systems and benefit from it."

Dustin M. Graham 